\title{
Communicating in a crisis: the H1N1 influenza pandemic
}

In 2009, with the influenza A (H1N1) virus, commonly known as swine flu, the world experienced the first influenza pandemic of the 21st century and the first for 40 years. For the vast majority of GPs and other health professionals, this has been the first experience of pandemic flu in our professional lifetimes. Compared to the influenza pandemics of the previous century, its impact has been relatively mild. However, it has been bad enough, with over 400 deaths in the UK and many thousands of people having been admitted to hospital or to critical care units. In the UK, we were fortunate that the pandemic waves occurred over the summer and autumn rather than at the time of peak winter activity in the NHS, but even so services were hard pressed in general and many general practices and primary care out-of-hours providers, especially in 'hot-spot areas', found they were stretched to the limit.

Given that there are no guarantees that it will be 40 years or so until the next influenza pandemic and also that a future pandemic virus might well be more virulent, it is imperative that professionals and policy makers examine our recent experience and identify what went well, and more importantly, what needs to be improved to allow us to best meet the challenges of the next flu pandemic, or other major public health crisis.

One area for reflection is that of professional communications. The article by Caley et al illustrates the difficulties experienced by GPs in the West Midlands in dealing with communications from a variety of sources with sometimes inconsistent advice. ${ }^{1}$ The problem here appeared to be too much information, from too many sources. The potential problem of too little information, or information from non-trusted sources, was not identified by the responders to the study. As these responders were practice-based doctors, neither was the issue of lack of communications to some important groups addressed. For instance, in my role as pandemic lead for the Royal College of General Practitioners (RCGP), I had many communications throughout the pandemic period from freelance GPs, who tended not to be included on local mailing lists. For many of these colleagues, their major source of information was that provided by national bodies, such as the RCGP.

In our previous work preparing for pandemic flu, ${ }^{2}$ colleagues from different agencies had appreciated the need for clear and direct communications to GPs and general practices. We anticipated that practices would need material targeted specifically at them and that this would need to be timely and from respected sources. The pandemic planners also acknowledged that although material may come from different sources, and from differing perspectives, it was important to avoid any elements of inconsistency that could lead to confusion and loss of confidence. The findings from the West Midlands study imply that these aims were not fully realised in practice. For the benefit of our successors, it is worth considering what factors played into this outcome and whether it might be possible to build on this experience with a view to greater coordination and consistency next time around.

It would be fair to say that the swine flu pandemic did not play out as had been anticipated in the work on pandemic planning that had been going on for several years. Public health information networks are always on the lookout for threats from influenza and other contagious illnesses and a number of 'candidate pandemic viruses' were being closely observed, including the feared H5N1 'bird flu' virus. However, the swine flu virus was known to very few experts, and for a very short time, prior to the first news reports emerging of a wave of young adults presenting with severe respiratory symptoms in Mexico City in late April 2009. Knowledge of the virology, epidemiology, and clinical features relating to this virus was established pretty well from scratch. Meanwhile, GPs in the UK, together with most healthcare workers worldwide, were dusting down pandemic plans and asking for pertinent information, almost in real-time.

National organisations such as the Health Protection Agency and the UK Departments of Health had important messages that they wanted to communicate to GPs, while professional organisations such as the RCGP wished to respond to requests from members for ongoing support. Locally, primary care organisations (PCOs) and local medical committees also had specific messages to convey to GPs and practices. It is easy to see from where the potential for multiple, and possibly conflicting, messages arises. The complaint of rapidly changing information is also valid, but this should be considered against the background of swiftly emerging information. The legal and regulatory framework also changed rapidly, in response to emerging evidence. GPs should understand that the reason for several different dosing regimens for antivirals in children was because of licensing changes by European regulators responding to developing evidence about use of these drugs. Drugs that had never before been used in a pandemic situation and where there was previously little evidence relating to use in babies and young children.

GPs might reasonably ask whether the different agencies talked to one another. In fact, a great deal of effort was put into organisational liaison, certainly at the national level, with frequent teleconferences, meetings, conversations, and a proliferation of e-mail correspondence. With the benefit of hindsight, it is reasonable to conclude that all this work might have been better coordinated, but as someone in the middle of it all at the time, I also believe that colleagues did well to communicate with each other as well as we actually did.

Currently work is underway, at both 
national and local level, to examine our recent experiences of the pandemic and there are certainly lessons to be learned. Although we had previously identified that communication to GPs and practices would be an important area, I don't believe anyone had identified in advance just how critical, or how difficult, it would turn out to be. In preparing for the future, I suggest that this is given even higher priority and supported with even greater resource. We should be able to separate out national and generic information from local and specific information in a more effective manner. Version control of documentation needs to be in place from the outset, and rigorously quality controlled. PCOs, in particular, need to consider how best to communicate with freelance GPs and other important services, such as out-of-hours providers.

Over the period of the pandemic, the RCGP issued frequent bulletins to its members and indeed put arrangements in place for these to be widely circulated so as to be available to as many GPs and practices as possible. This sometimes resulted in some doctors receiving the same message more than once. In fact the College took a view that it would be better for some GPs to receive duplicated information than for some not to receive it at all. It is possible that more sophisticated methods of communication might allow for more highly-targeted distribution in the future. Nonetheless, despite all the identified problems, the feedback received by the College was that the information provided was well regarded and greatly appreciated by GPs.

We have the opportunity now to reflect on this experience. Next time - and there will be a next time - the threat could be more dangerous and the challenge for general practice even greater. Professional organisations like the RCGP and British Medical Association; local and national organisations; and the UK Departments of Health all need to actively review their plans for professional communications and learn the lessons from swine flu in 2009.

\section{Maureen Baker,}

Pandemic Lead for the Royal College of General Practitioners, London.

\section{Provenance}

Commissioned; not peer reviewed.

\section{REFERENCES}

1. Caley M, Sidhu K, Shukla R. GPs' opinions on the NHS and HPA response to the first wave of the influenza A/H1N1v pandemic. Br J Gen Pract 2010; 60: 283-285.

2. Royal College of General Practitioners, British Medical Association's General Practitioners Committee. Preparing for pandemic influenza. Guidance for practices. What to do now and in a pandemic.

http://www.rcgp.org.uk/pdf/gp_guidance.pdf (accessed 10 Mar 2010).

DOI: 10.3399/bjgp10X483850

\section{ADDRESS FOR CORRESPONDENCE}

\section{Maureen Baker}

Pandemic Lead, Royal College of General Practitioners, 14 Princes Gate, Hyde Park, London SW7 1PU.

Email: mbaker@rcgp.org.uk 